\title{
The Boundedness Problem for Monadic Universal First-Order Logic
}

\author{
Martin Otto \\ Fachbereich Mathematik \\ Technische Universität Darmstadt \\ 64289 Darmstadt, Germany
}

\begin{abstract}
We consider the monadic boundedness problem for least fixed points over FO formulae as a decision problem: Given a formula $\varphi(X, x)$, positive in $X$, decide whether there is a uniform finite bound on the least fixed point recursion based on $\varphi$. Few fragments of $\mathrm{FO}$ are known to have a decidable boundedness problem; boundedness is known to be undecidable for many fragments. We here show that monadic boundedness is decidable for purely universal $\mathrm{FO}$ formulae without equality in which each non-recursive predicate occurs in just one polarity (e.g., only negatively). The restrictions are shown to be essential: waving either the polarity constraint or allowing positive occurrences of equality, the monadic boundedness problem for universal formulae becomes undecidable. The main result is based on a model theoretic analysis involving ideas from modal and guarded logics and a reduction to the monadic second-order theory of trees.
\end{abstract}

\section{Introduction}

Consider a formula $\varphi(X, x) \in \mathrm{FO}$ that is positive in the monadic second-order variable $X$ and with a single free first-order variable $x$. Over corresponding structures $\mathfrak{A}$ such $\varphi$ defines an operation on subsets,

$$
\begin{aligned}
F_{\varphi}^{\mathfrak{A}}: \mathcal{P}(A) & \longrightarrow \mathcal{P}(A) \\
P & \longmapsto\{a \in A: \mathfrak{A} \models \varphi[P, a]\} .
\end{aligned}
$$

Due to positivity in $X$, this operation is monotone w.r.t. $\subseteq$, and hence has a least fixed point $\left(\mu_{X} \varphi\right)^{\mathfrak{A}}$, which is also attained as the limit of the monotone sequence of its stages $X^{\alpha}[\mathfrak{A}]$ generated by iteration of $F_{\varphi}^{\mathfrak{A}}$ on $\emptyset$.

When is this recursion uniformly finitely bounded, and hence the least fixed point uniformly definable by a finite iteration of $\varphi$ ? We say that $\varphi(X, x)$ is bounded iff there is $n \in \mathbb{N}$ such that $X^{n+1}[\mathfrak{A}]=X^{n}[\mathfrak{A}]\left(=\left(\mu_{X} \varphi\right)^{\mathfrak{A}}\right)$ for all $\mathfrak{A}$. We consider the boundedness problem as a decision prob- lem $\operatorname{Bdd}(\mathcal{F})$, for formulae $\varphi$ from a fixed syntactic fragment $\mathcal{F} \subseteq$ FO.

$$
\begin{array}{l|l}
\operatorname{Bdd}(\mathcal{F}) \quad \begin{array}{l}
\text { given } \varphi=\varphi(X, x) \in \mathcal{F} \\
\text { decide if } \varphi \text { is bounded }
\end{array}
\end{array}
$$

This decision problem has originally been considered in particular for the query language Datalog with a view to query optimisation $[1,13,9,11,12]$. However, the overwhelmingly negative picture w.r.t. decidability there has largely defeated initial hopes that bounded (i.e., spurious) recursion could be systematically detected and eliminated.

But similar concerns arise also for instance in connection with temporal fixed point logics like the $\mu$-calculus, cf. Theorem 2 below, or other fixed point formalisms. Because of the model theoretic link between boundedness and FO definability of the least fixed point, there is also a connection with circumscription, which is of interest in artificial intelligence [17, 16]. By the Barwise-Moschovakis theorem [5] (cf. Theorem 6 below), $\varphi$ is bounded if, and only if, $\mu_{X} \varphi$ is FO definable. So, for circumscriptions based on FO formulae that are positive in the target predicate, FO definability of this target predicate is the same as boundedness.

Overall however, very few fragments of FO are known to have a decidable boundedness problem. Quite to the contrary, boundedness is known to be undecidable for many fragments. Among the scarce decidability results, the following result of Cosmadakis, Gaifman, Kanellakis, Vardi [9] stands out.

Theorem 1. Boundedness is decidable for monadic Datalog. I.e., Bdd is decidable for positive existential $\mathrm{FO}$ formulae in monadic recursion variables $X$, as well as for systems of such.

Another decidability result that is important for our considerations is the following from [19] (cf. the modal variant of Theorem 6 below).

Theorem 2. $\mathrm{Bdd}(\mathrm{ML})$, the boundedness problem for the modal fragment of FO is decidable. More generally, one 
can decide for formulae of the $\mu$-calculus whether they are equivalent to plain modal formulae.

Undecidability results for Bdd abound; we mention some. See [12] for (i), [11] for (ii), and [14] for (iii). For (iv) and (v) compare section 3.2 below.

Theorem 3. $\operatorname{Bdd}(\mathcal{F})$ is undecidable for the following fragments $\mathcal{F}$ of $\mathrm{FO}$ :

(i) $\mathcal{F}$ : positive existential formulae in a binary recursive predicate $X$.

(ii) $\mathcal{F}$ : existential formulae in a monadic recursive predicate if negation (or even just inequality) is allowed.

(iii) $\mathcal{F}$ : formulae in a monadic recursive predicate using just two distinct first-order variables.

(iv) $\mathcal{F}$ : universal formulae in a monadic recursive predicate, negative in all non-recursive predicates but allowing positive occurrences of equality.

(v) $\mathcal{F}$ : universal $\mathrm{FO}$ formulae in a monadic recursive predicate, if some of the non-recursive relations can occur both positively and negatively.

In a way, therefore, few natural fragments remain that would be candidates for a decidable boundedness problem. Among the most obvious are: the guarded fragment (still open) and the universal fragment of FO (settled here). The present investigation shows that monadic boundedness is decidable for purely universal FO formulae without (positive occurrences of) equality in which each non-recursive predicate occurs in just one polarity. As the boundedness issue is obviously invariant under swapping relations for their complements in the underlying relational structures, we may w.l.o.g. restrict attention to the case where all nonrecursive predicates (and equality) only appear negatively, denoted by $\mathcal{F}(\forall,-)$ in the following.

Main Theorem $\operatorname{Bdd}(\mathcal{F}(\forall,-))$ is decidable. I.e., boundedness is decidable for monadic recursion over purely universal FO formulae that are purely negative in all nonrecursive predicates and equality (or, by extension, universal formulae in which each predicate letter occurs in just one polarity).

The restrictions expressed in the theorem are essential. The decidability result obtained provides a counterpart to the one expressed in Theorem 1. Note, however, that the logical "dual" of the decidability result in Theorem 1 would concern boundedness for greatest fixed points of universal formulae, rather than least fixed points. Conversely, our main theorem proves, by duality, decidability of the boundedness problem for greatest fixed points of purely positive, purely existential formulae.

Organisation. In the following section, we review the basic definitions and discuss the model theoretic connection between boundedness and definability of the fixed point (Barwise-Moschovakis theorem). Section 3 focuses on boundedness for the universal fragment of FO and introduces a normal form for universal fixed points; we also contrast our decidability result with undecidability proofs for less restricted classes of universal formulae. In section 4 we prepare the reduction argument underlying our main result by collecting the relevant facts about decidability of boundedness for systems in the modal fragment; we also give a new model theoretic argument for decidability there. The main technical contribution in section 5 provides the reduction from the restricted universal case to the modal case (based on an intuitive intermediate stage that is motivated by a formalisation in the guarded fragment). We conclude with some remarks on ramifications and open problems in section 6 .

\section{Preliminaries}

\subsection{Boundedness}

Consider fixed point recursion in systems for simultaneous least fixed points. (Even when we are ultimately interested in boundedness for single formulae rather than systems, the reductions applied take us to systems.)

An admissible system is given by a tuple of FO formulae $\bar{\varphi}=\left(\varphi_{1}, \ldots, \varphi_{k}\right)$ in a relational vocabulary $\tau$ and monadic second-order variables $\bar{X}=\left(X_{1}, \ldots, X_{k}\right)$, where each $\varphi_{i}$ is of the form

$$
\varphi_{i}(\bar{X}, x) \in \mathrm{FO}, \text { positive in each } X_{j} .
$$

Over any $\tau$-structure $\mathfrak{A}$ associate with this system the monotone operator on $(\mathcal{P}(A))^{k}$

$$
\bar{P}=\left(P_{i}\right)_{1 \leqslant i \leqslant k} \longmapsto\left(\left\{a \in A: \mathfrak{A}=\varphi_{i}[\bar{P}, a]\right\}\right)_{1 \leqslant i \leqslant k} .
$$

The simultaneous least fixed point of this operator is denoted $\bar{\varphi}^{\infty}[\mathfrak{A}]$; its components $\left(\bar{\varphi}^{\infty}[\mathfrak{A}]\right)_{i}$. The least fixed point $\bar{\varphi}^{\infty}$ is also the limit $\bar{X}^{\infty}$ of the monotone sequence of stages $\bar{X}^{\alpha}[\mathfrak{A}]=\bar{X}^{\alpha}=\left(X_{1}^{\alpha}, \ldots, X_{k}^{\alpha}\right)$ inductively defined over the given $\mathfrak{A}$ according to

$$
\begin{aligned}
X_{i}^{0} & =\emptyset \\
X_{i}^{\alpha+1} & =\left\{a \in A: \mathfrak{A}=\varphi_{i}[\bar{P}, a]\right\} \\
X_{i}^{\lambda} & =\bigcup_{\alpha<\lambda} X_{i}^{\alpha} \text { for limit } \lambda .
\end{aligned}
$$

The closure ordinal $\gamma[\bar{\varphi}, \mathfrak{A}]$ of $\bar{\varphi}$ in $\mathfrak{A}$ is the least $\alpha$ such that $\bar{X}^{\alpha}[\mathfrak{A}]=\bar{\varphi}^{\infty}[\mathfrak{A}]$.

Observation 4. Over $\omega$-saturated $\mathfrak{A}$, the closure ordinal for any first-order least fixed point is bounded by $\omega$.

Note that all finite stages $X_{i}^{n}$, for $n \in \mathbb{N}$, are uniformly FO definable. We write $\varphi^{n}$ for the formula that defines the $n$-th stage of $\varphi$, which is obtained inductively by substituting $\varphi^{n-1}$ for $X$ in $\varphi(X, x)$. 
Definition 5. The admissible system $\bar{\varphi}=\left(\varphi_{1}, \ldots, \varphi_{k}\right)$ is bounded if for some $n \in \mathbb{N}, \gamma[\bar{\varphi}, \mathfrak{A}] \leqslant n$ for all $\mathfrak{A}$. This notion naturally relativise to restricted classes $\mathcal{C}$ of structures $\mathfrak{A}$; we speak of boundedness of $\bar{\varphi}$ over $\mathcal{C}$ in this sense.

Unboundedness as generalised satisfiability. It is useful to think of unboundedness as a satisfiability issue in the following sense. $\varphi(X, x)$ is unbounded iff $\varphi^{n+1}(x) \wedge \neg \varphi^{n}(x)$ is satisfiable for all $n \in \mathbb{N}$. It is not hard to see that, for fragments satisfying some mild closure properties, $\operatorname{SAT}(\mathcal{F})$ is reducible to $\operatorname{Bdd}(\mathcal{F})$.

Boundedness in finite model theory. For many natural fragments of FO, boundedness (over all structures) coincides with boundedness over the class of all finite structures. This is easily seen for fragments that are closed under positive substitution, negation and conjunction and that have the finite model property. Closure under positive substitution implies that the finite stages $X^{n}$ in the fixed point generation are themselves definable by formulae $\varphi^{n}$ in the given fragment $\mathcal{F}$; closure under conjunction and negation provide the formulae $\varphi^{n+1} \wedge \neg \varphi^{n}$ within $\mathcal{F}$. For such fragments unboundedness (in infinite structures) implies unboundedness over finite structures, by an appeal to the finite model property. This applies in particular to the modal, guarded and two-variable fragments.

For the existential and universal fragments, one can argue similarly, but invoking the finite model property for the $\exists^{*} \forall^{*}$ fragment of FO (the Bernays-Schönfinkel class of the classical decision problem, see [8]) for $\varphi^{n+1} \wedge \neg \varphi^{n}$.

\subsection{The BarwiseMoschovakis Theorem}

The following is an adaptation of a well-known classical theorem [5], which links boundedness to FO definability of the fixed point. The non-obvious part, (iii) $\Rightarrow$ (i), is based on a compactness argument using $\omega$-saturated structures $\mathfrak{A}$, applying Observation 4.

Theorem 6 (Barwise-Moschovakis). For admissible $\bar{\varphi}$, the following are equivalent:

(i) $\bar{\varphi}$ is bounded.

(ii) $\left(\bar{\varphi}^{\infty}\right)$ is uniformly $\mathrm{FO}$ definable over all $\mathfrak{A}$.

(iii) $\left(\bar{\varphi}^{\infty}[\mathfrak{A}]\right)$ is FO definable for each $\mathfrak{A}$.

The statement of the theorem relativises to elementary classes. It also relativises to fragments $\mathcal{F} \subseteq$ FO with the property that the finite stages of admissible systems over $\mathcal{F}$ are themselves $\mathcal{F}$-definable. Natural fragments of this kind are, for instance, the universal or the existential fragments of $\mathrm{FO}$, the $k$-variable fragments $\mathrm{FO}^{k}$, the guarded fragment GF and modal logic ML. For the latter, for instance, we have that an admissible system $\bar{\varphi}$ of modal formulae is bounded if, and only if, its least fixed point is definable in modal logic (over each individual Kripke structure).
Status in finite model theory. As discussed in the previous section, the boundedness problem coincides with its finite model theory version for several 'good' fragments, including the universal fragment. For the finite model theory (fmt) version of the Barwise-Moschovakis theorem, however, one also needs to link definability of the fixed point over all finite structures to boundedness over all finite structures, and hence (for a 'good' fragment) to boundedness and therefore definability of the fixed point over all structures. For the existential positive fragment, a strong form of the finite model theory version of the Barwise-Moschovakis theorem was proved by Ajtai and Gurevich: a Datalog query is FO definable if, and only if, the program is bounded. This result has recently been put in a new context, by major new results on the fmt version of the classical preservation theorem that links preservation under homomorphisms to positive existential definability, by Rossmann [21] and Atserias, Dawar and Kolaitis [4]. These fmt versions show that FO definability of the fixed point of a positive existential recursion implies its positive existential definability, due to preservation of the fixed point under homomorphisms in finite structures. By duality, Rossmann's theorem similarly implies that FO definability for a universal equality-free recursion that is negative in all non-recursive predicates implies its universal FO definability, in all finite structures.

For the existential fragment, one can show directly that existential FO definability of the fixed point over all finite structures implies its definability (by the same existential formula) over all structures, again by use of the finite model property of the $\exists^{*} \forall^{*}$ fragment. It is unclear, though, whether also for the universal fragment, universal definability of the fixed point in all finite structures implies its definability over all structures.

\section{Universal FO}

The formulae of the universal fragment of FO are generated from atomic and negated atomic formulae by conjunction, disjunction and universal quantification.

\subsection{Moschovakis normal form}

The following normal form for universal formulae $\varphi(X, x)$ that are positive in the second-order variable $X$ is an adaptation of the normal form of [18], Chapter 4B.

\section{Proposition 7 (Moschovakis).}

Let $\varphi(X, x)$ positive in $X$ and purely universal. Then there is a formula $\tilde{\varphi}(X, x)$ of the form

$$
\tilde{\varphi}(X, x)=\forall \mathbf{y}(\theta(x, \mathbf{y}) \rightarrow \delta(X, \mathbf{y})),
$$

where $\theta$ is quantifier free and does not contain $X$, and where $\delta$ is a disjunction of atoms $X y_{j}$ for some $y_{j} \in \mathbf{y}$ 
such that

$$
\neg \forall x X x \models \forall x(\varphi(X, x) \leftrightarrow \tilde{\varphi}(X, x))
$$

If $\varphi$ is purely negative in all non-recursive predicates and in $=$, then $\theta$ can be chosen purely positive (in all predicates and in $=$ ).

Proof. We sketch the key steps in the inductive proof.

For $\varphi=X z$, let $\tilde{\varphi}:=\forall y(y=z \rightarrow X y)$.

For $\varphi=R \mathbf{z}$, let $\tilde{\varphi}:=\forall y(\neg R \mathbf{z} \rightarrow X y)$. Here equivalence with $\varphi$ relies on the existence of elements outside $X$.

The universal quantification step is trivial. The interesting inductive steps are for $\wedge$ and $\vee$ :

Consider $\varphi=\varphi_{1}(X, \mathbf{z}) * \varphi_{2}(X, \mathbf{z})$, where $* \in\{\wedge, \vee\}$. Assume that $\tilde{\varphi}_{i}=\forall \mathbf{y}^{(i)}\left(\theta_{i}\left(\mathbf{x}, \mathbf{y}^{(i)}\right) \rightarrow \delta_{i}\left(X, \mathbf{y}^{(i)}\right)\right)$ is as desired for $\varphi_{i}$, w.l.o.g. with disjoint $\mathbf{y}^{(i)}$. Let $\tilde{\varphi}$ be the following, where $\bar{*}$ is the dual of $*$ :

$$
\forall \mathbf{y}^{(1)} \forall \mathbf{y}^{(2)}\left[\begin{array}{l}
\left(\left(\theta_{1}\left(\mathbf{z}, \mathbf{y}^{(1)}\right) \bar{*} \theta_{2}\left(\mathbf{z}, \mathbf{y}^{(2)}\right)\right)\right. \\
\rightarrow\left(\delta_{1}\left(X, \mathbf{y}^{(1)}\right) \vee \delta_{2}\left(X, \mathbf{y}^{(2)}\right)\right)
\end{array}\right]
$$

In both cases $(*=\wedge$ or $*=\vee)$ it is clear that $\varphi(X, \mathbf{z}) \models$ $\tilde{\varphi}(X, \mathbf{z})$. It remains to establish, that, whenever $X$ is not the full universe, then $\tilde{\varphi}$ implies $\varphi$.

Consider first the case of $*=\vee$. We look at a model of $\psi$, and suppose for instance that $\forall \mathbf{y}^{(1)}\left[\theta_{1}\left(\mathbf{y}^{(1)}\right) \rightarrow\right.$ $\left.\delta_{1}\left(X, \mathbf{y}^{(1)}\right)\right]$ does not hold true. We therefore find an interpretation for $\mathbf{y}^{(1)}$ such that $\theta_{1} \wedge \neg \delta_{1}$; fixing any such interpretation for $\mathbf{y}^{(1)}, \psi$ becomes equivalent to $\forall \mathbf{y}^{(2)}\left[\theta_{2}\left(\mathbf{y}^{(2)}\right) \rightarrow \delta_{2}\left(X, \mathbf{y}^{(2)}\right)\right]$, as it should.

Consider now the case of $*=\wedge$. Again, we look at a model of $\psi$, in which there are elements outside $X$. We need to show that, for instance, this implies that $\theta_{1}\left(\mathbf{y}^{(1)}\right) \rightarrow$ $\delta_{1}\left(X, \mathbf{y}^{(1)}\right)$. Instantiate the universally quantified $\mathbf{y}^{(2)}$ in $\psi$ by a tuple consisting of components not in $X$; this makes $\delta_{2}$ false. Then $\psi$ implies that $\theta_{1}\left(\mathbf{y}^{(1)}\right) \rightarrow \delta_{1}\left(X, \mathbf{y}^{(1)}\right)$, as desired.

The normal form given in [18] goes further in also eliminating the disjunctions in $\delta$ in favour of existential quantification in the prefix; this is not desirable here.

\subsection{Limits for decidability}

The following is in marked contrast with our main decidability result.

Theorem 8. Bdd is undecidable for monadic recursion w.r.t. purely universal formulae in which all non-recursive predicates occur only negatively, but equality may occur positively.
The proof follows a general pattern established in [14] and in [15] for several other undecidable cases of the monadic boundedness problem, via reduction of the tiling problem [7]. A domino system is a finite structure $\mathfrak{D}=$ $\left(D, R_{H}, R_{V}\right)$ consisting of tile types $d \in D$ with two adjacency relations $R_{H}$ and $R_{V}$ that specify when two tile types fit in horizontally or vertically adjacent positions in tiling.

Formally a $\mathfrak{D}$-tiling of $n \times n$ is a homomorphism from the $n \times n$ grid structure $\mathfrak{G}_{n}$ to $\mathfrak{D}$. Here

$$
\begin{aligned}
\mathfrak{G}_{n} & =\left(\{0, \ldots, n-1\}^{2}, H, V\right) \\
\text { with } H & =\{((i, j),(i+1, j)): i+1, j<n\}, \\
V & =\{((i, j),(i, j+1)): i, j+1<n\} .
\end{aligned}
$$

Intuitively, a $\mathfrak{D}$-tiling of $n \times n$ corresponds to a placement of tile types on the nodes of the $n \times n$ grid such that horizontally and vertically adjacent tiles respect the adjacency constraints given by $R_{H}$ and $R_{V}$, respectively. We also refer to a corresponding expansion of $\mathfrak{G}_{n}$ by colours $\left(P_{d}\right)_{d \in D}$ as a $\mathfrak{D}$-tiling of $n \times n$.

Theorem 9 (Berger). The following problem is undecidable: Given a tiling system $\mathfrak{D}$, decide whether $\mathfrak{D}$ admits tilings of size $n$ for arbitrarily large $n \in \mathbb{N}$.

We now want to associate with a given $\mathfrak{D}$ a formula $\varphi_{\mathfrak{D}}=\varphi(X, x)$, positive in $X$, purely universal and with only negative occurrences of the relations $H, V$ (binary) and $P_{d}$ (unary) for $d \in D$, such that $\varphi_{\mathfrak{D}}$ is unbounded iff $\mathfrak{D}$ admits arbitrarily large tilings. Consider firstly an auxiliary sentence $\varphi_{0}$ which is the conjunction of the following:

$$
\begin{aligned}
& \forall y \bigwedge_{d \neq d^{\prime}} \neg\left(P_{d} y \wedge P_{d^{\prime}} y\right) \\
& \forall y_{1} \forall y_{2} \neg\left(H y_{1} y_{2} \wedge \bigvee_{\left(d, d^{\prime}\right) \notin R_{H}}\left(P_{d} y_{1} \wedge P_{d^{\prime}} y_{2}\right)\right) \\
& \forall y_{1} \exists y_{2} \neg\left(V y_{1} y_{2} \wedge \bigvee_{\left(d, d^{\prime}\right) \notin R_{V}}\left(P_{d} y_{1} \wedge P_{d^{\prime}} y_{2}\right)\right) \\
& \forall y y_{1} y_{2}\left(\left(H y y_{1} \wedge H y y_{2}\right) \rightarrow y_{1}=y_{2}\right) \\
& \forall y y_{1} y_{2}\left(\left(V y y_{1} \wedge V y y_{2}\right) \rightarrow y_{1}=y_{2}\right) \\
& \forall y \forall y_{1} \forall y_{2} \forall z_{1} \forall z_{2}\left[\begin{array}{r}
\left(H y y_{1} \wedge V y_{1} z_{1} \wedge V y y_{2} \wedge H y_{2} z_{2}\right) \\
\\
\rightarrow z_{1}=z_{2}
\end{array}\right]
\end{aligned}
$$

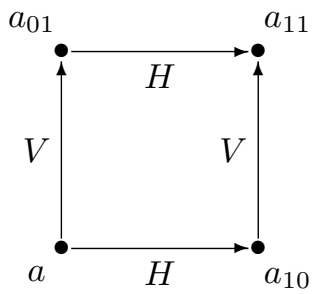

$\varphi=\varphi_{\mathfrak{D}}$ will be of the form $\varphi(X, x)=\varphi_{0} \wedge \varphi_{1}(X, x)$ with static part $\varphi_{0}$ as given above. Clearly $\varphi$ is unbounded iff $\varphi_{1}$ is unbounded in models of $\varphi_{0}$. Models of $\varphi_{0}$ are $H$-V-graphs in which $H$ and $V$ are the graphs of partial 
functions that commute where defined, and in which each node carries at most one colour $P_{d}$ in such a way that the colours of adjacent nodes respect the tiling constraints.

$\varphi_{1}$ is set up so as to ensure that (in models of $\varphi_{0}$ ) the recursion on $\varphi_{1}(X, x)$ has closure ordinal $n$ on $\mathfrak{D}$-tilings of $\mathfrak{G}_{n}$, and that any structure on which $\varphi_{1}$ has a non-trivial $(n+1)$-st stage, embeds $\mathfrak{G}_{n}$ and hence establishes the existence of a $\mathfrak{D}$-tiling of $n \times n$.

The formalisation of $\varphi_{1}$ uses the following abbreviations (corresponding to positive quantifier-free definitions):

$$
\begin{aligned}
\dot{H}:= & \left\{(x, y): H x y \wedge \bigvee_{d \in D} P_{d} y\right\}, \\
\dot{V}:= & \left\{(x, y): V x y \wedge \bigvee_{d \in D} P_{d} y\right\} . \\
\text { Put } \varphi_{1}(X, x):= & \forall y(\dot{H} x y \rightarrow X y) \\
& \vee \quad \forall y(\dot{V} x y \rightarrow X y) \\
& \vee \quad \forall y \forall z((\dot{H} x y \wedge \dot{V} y z) \rightarrow X z) \\
& \vee \quad \forall y \forall z((\dot{V} x y \wedge \dot{H} y z) \rightarrow X z) .
\end{aligned}
$$

Obviously $\varphi_{\mathfrak{D}}:=\varphi_{0} \wedge \varphi_{1}(X, x)$ is purely negative in $H, V$ and the $P_{d}$, but uses equality positively (in $\varphi_{0}$ ).

It is obvious that $\gamma\left[\varphi_{\mathfrak{D}}, \mathfrak{A}\right]=n$ for a $\mathfrak{D}$-tiling $\mathfrak{A}=$ $\left(\mathfrak{G}_{n},\left(P_{d}\right)\right)$ of $n \times n$. It remains to show that conversely, unboundedness of $\varphi_{\mathfrak{D}}$ implies that $\mathfrak{D}$ has tilings of arbitrarily large $n \times n$ grids.

Lemma 10. Let $\mathfrak{A} \models \varphi_{0}, X^{i}[\mathfrak{A}]$ the stages of $\varphi_{\mathfrak{D}}$ on $\mathfrak{A}$. Let $\dot{\mathfrak{A}}$ be $\mathfrak{A}$ with $H$ and $V$ replaced by $\dot{H}$ and $\dot{V}$, respectively. Let a $\notin X^{n}[\mathfrak{A}]$. Then there is a homomorphism $h: \mathfrak{G}_{n+1} \rightarrow \dot{\mathfrak{A}}$ with $h(0,0)=a$. It follows that there is a $\mathfrak{D}$-tiling of $\mathfrak{G}_{n}$ if $\gamma\left[\varphi_{\mathfrak{D}}, \mathfrak{A}\right]>n$.

Proof. The main claim is proved by induction. Note that $a \notin X^{n}[\mathfrak{A}]$ implies that $a$ has $\dot{H}-, \dot{V}$ - and $(\dot{V} \circ \dot{H})$ - and $(\dot{H} \circ \dot{V})$-successors $a_{10}, a_{01}, a_{11}$ and $a_{11}^{\prime}$ that are not in $X^{n-1}[\mathfrak{A}] . \quad \varphi_{0}$ forces $a_{11}=a_{11}^{\prime}$. By the inductive hypothesis there are homomorphisms $h_{i}: \mathfrak{G}_{n} \rightarrow \dot{\mathfrak{A}}$ with $h_{1}(0,0)=a_{10}, h_{2}(0,0)=a_{01}$ and $h_{3}(0,0)=a_{11}$.

$\varphi_{0}$ now also forces $h_{1}(0,1)$ (the $V$-successor of $a_{10}$ ) to be the same as $h_{2}(1,0)$ (the $H$-successor of $\left.a_{01}\right)$ and $h_{3}(0,0)$. Similarly, $h_{1}(i, j+1)=h_{2}(i+1, j)=h_{3}(i, j)$ for all $i, j$ where defined. But this implies that these three homomorphisms can be combined consistently to obtain the desired homomorphism $h$ by putting $h(0,0):=a$, $h(i+1, j):=h_{1}(i, j), h(i, j+1):=h_{2}(i, j)$ and $h(i+1, j+1):=h_{3}(i, j)$ (whichever righthand sides are defined).

Instead of equality one can introduce an extra binary relation $\approx$ which, by purely universal axioms, can be forced to be a congruence w.r.t. $H, V$ and the $P_{d}$. However, these axioms will necessarily involve $\approx$ and the predicates $H, V$, $P_{d}$ in both polarities. In other words, we may trade the restriction on polarities for avoidance of equality.

Theorem 11. Bdd is undecidable for monadic recursion w.r.t. purely universal formulae without equality.

\section{Boundedness in modal logic}

We regard modal logic as a fragment $\mathrm{ML} \subseteq$ FO in the usual way. In a vocabulary $\sigma$ consisting of binary relations $E_{i}$ and unary predicates $P_{j}$, the formulae of $\operatorname{ML}[\sigma]$ are generated as the closure of the atomic formulae $P_{j}$ under booleans and modal quantification $\left\langle E_{i}\right\rangle$ and $\left[E_{i}\right]$. In FO terms, $\left(\left\langle E_{i}\right\rangle \varphi\right)(x)$ is $\exists y\left(E_{i} x y \wedge \varphi(y)\right)$, and dually $\left(\left[E_{i}\right] \varphi\right)(x)$ is $\forall y\left(E_{i} x y \rightarrow \varphi(y)\right)$.

We consider admissible systems in a tuple of monadic recursive predicates $\bar{X}$. The modal version of the BarwiseMoschovakis theorem provides the means to decide boundedness, ultimately via reduction to the MSO theory of the $\omega$-branching tree, which is decidable by Rabin's well known theorem. We outline the chain of model theoretic arguments underlying this reduction.

Due to bisimulation invariance and the LöwenheimSkolem theorem, it suffices to consider the behaviour of systems in ML over countable tree structures.

Let $\sigma$ be a relational type consisting of binary predicates $E_{i}$ (for labelled edges) and unary predicates $P_{j}$ (for colouring or labelling vertices). A tree structure of type $\sigma$ is a rooted directed tree w.r.t. $E:=\bigcup E_{i}$ with disjoint $E_{i}$ (no multiple edge labels).

For a tree structure $\mathfrak{A}, \lambda$ with root $\lambda$ and $\ell \geqslant 1$ we let $A\lceil\ell \subseteq A$ denote the initial segment consisting of all nodes up to depth $\ell$. Some related terminology:

(a) $A^{\prime} \subseteq A$ is called initial in $\mathfrak{A}, \lambda$ if it contains the root $\lambda$ and is connected.

(b) $A^{\prime} \subseteq A$ is called bounded if $A^{\prime} \subseteq A\lceil\ell$ for some $\ell \in \mathbb{N}$.

(c) $A^{\prime} \subseteq A$ is called path-finite if it contains no infinite paths.

Note that, while properties (a) and (c) are MSO definable, property (b) is not.

Definition 12. Call a formula $\psi(x) \in \mathrm{MSO}$ tree-local if there is some $\ell \in \mathbb{N}$ such that for all countable tree structures $\mathfrak{A}$ and for all initial subsets $A^{\prime} \supseteq A\lceil\ell$ :

$$
\mathfrak{A}, \lambda \models \psi \quad \Leftrightarrow \quad \mathfrak{A}\left\lceil A^{\prime}, \lambda \models \psi .\right.
$$

The following is a straightforward consequence of the combination of the modal variant of Theorem 6 and the expressive completeness of ML for bisimulation invariant properties that are $\ell$-local for some $\ell[6,20]$.

Lemma 13. The following are equivalent for admissible systems $\bar{\varphi} \in \mathrm{ML}$ :

(i) $\bar{\varphi}$ bounded.

(ii) $\bar{\varphi}^{\infty}$ tree-local.

(iii) $\bar{\varphi}^{\infty}$ ML-definable.

Theorem 14. $\operatorname{Bdd}(\mathrm{ML})$ is decidable via reduction to the MSO theory of the $\omega$-branching tree $\mathfrak{T}_{\omega}$. Similarly for 
boundedness of modal systems over MSO definable classes of trees.

The proof of correctness for the intended reduction involves a regular version of König's lemma that allows us to "apply König's lemma" in the context of the $\omega$-branching tree, its infinite branching notwithstanding.

A regular expansion of $\mathfrak{T}_{\omega}$ is one that realises only finitely many isomorphism types of subtrees.

Lemma 15. The following are equivalent for initial subsets $D$ in regular expansions $\left(\mathfrak{T}_{\omega}, D\right)$ :

(i) D is path-finite.

(ii) $D$ is bounded.

Proof. For (i) $\Rightarrow$ (ii) one shows that one can inductively choose an infinite path within an unbounded $D$. Starting from the root, select a successor node in $D$ such that $D$ is unbounded in restriction to the subtree rooted in that node. This is always possible, as there are only finitely many isomorphism types of subtrees rooted in the available successor nodes. So $D$ cannot be bounded in each of those without being bounded in the father node.

Let

$$
\psi(Z):=\forall \bar{I}\left(\psi_{0}(\bar{I}) \rightarrow \bigwedge_{i}\left(\varphi(\bar{I})_{i}^{\infty} \leftrightarrow \varphi(\bar{I} \mid Z)_{i}^{\infty}\right)\right)
$$

be an MSO formula that says (of an initial subset $Z$ of $\mathfrak{T}_{\omega}$ ) that for all interpretations of countable tree structures of type suitable for $\bar{\varphi}$, the evaluation of the fixed point produces the same outcome at the root whether $\bar{\varphi}$ is evaluated in the whole structure or in the initial substructure induced on $Z$. Here $\psi_{0}(\bar{I})$ collects the obvious FO conditions on unary predicates $\bar{I}$ to encode an interpretation of a countable tree structure of the appropriate type within $\mathfrak{T}_{\omega}$ in the natural way. The intended interpretations are such that all nodes at depth $\ell$ in the interpreted tree are represented by depth $\ell$ nodes in $\mathfrak{T}_{\omega}$. This compatibility with depth is crucial for the reduction; it is for this reason that we work with the $\omega$-branching tree rather than the binary tree.

Lemma 16. The following are equivalent:

(i) $\bar{\varphi}$ bounded.

(ii) for some $\ell \in \mathbb{N}$ and for $D=T_{\omega}\left\lceil\ell: \mathfrak{T}_{\omega}, \lambda \models \psi[D]\right.$.

(iii) $\mathfrak{T}_{\omega}, \lambda \models \exists Z(Z$ initial and path-finite $\wedge \psi(Z))$.

(iv) there is some regular expansion $\left(\mathfrak{T}_{\omega}, D\right)$ of $\mathfrak{T}_{\omega}$ with an initial, path-finite $D$, such that $\mathfrak{T}_{\omega}, \lambda \models \psi[D]$.

Note that (iii) is decidable, as part of the MSO theory of $\mathfrak{T}_{\omega}$, thus proving the theorem.

Proof. (i) $\Rightarrow$ (ii) $\Rightarrow$ (iii) are clear. (iii) $\Rightarrow$ (iv) is a well known fact about MSO: any MSO formula satisfiable in some tree model has a regular tree model. (iv) $\Rightarrow$ (i) follows with Lemma 15.

\section{From modal to universal boundedness}

Consider boundedness for an equality-free universal formula $\varphi(X, x)$, positive in $X$, and with all other predicates of one polarity only. W.l.o.g. assume that $\varphi$ is in Moschovakis normal form (see Proposition 7) and purely negative in all predicates other than $X$.

$$
\begin{aligned}
\varphi(X, x) & =\forall \mathbf{y}\left(\left(\bigvee_{i} \theta_{i}(x, \mathbf{y})\right) \rightarrow \delta(X, \mathbf{y})\right) \\
& \left.\equiv \bigwedge_{i=1}^{k} \forall \mathbf{y}\left(\theta_{i}(x, \mathbf{y})\right) \rightarrow \delta(X, \mathbf{y})\right)
\end{aligned}
$$

the $\theta_{i}$ conjunctions of relational atoms (and not involving $X), \delta$ a disjunction over $X$-atoms in variables $\mathbf{y}$.

Intuitively we treat the $\theta_{i}$ as if they were relational guards (in the sense of the guarded fragment, [3]). To this end, we use new relation symbols $R_{i}$, whose intended interpretations are the relations defined by $\theta_{i}$. We may also assume w.l.o.g. that all these $R_{i}$ have the same arity $m+1$ where $m$ is the arity of $\mathbf{y}$. Let $\tau$ be the original vocabulary of $\varphi, \tau^{\theta}$ its extension by these $R_{i}$.

With $\tau$-structures $\mathfrak{A}$ we associate corresponding expansions to $\tau^{\theta}$-structures $\mathfrak{A}^{\theta}:=(\mathfrak{A}, \bar{R})$ by $R_{i}$ defined by $\theta_{i}$ according to

$$
R_{i}^{\mathfrak{A}}:=\left\{\mathbf{a}=\left(a_{0}, a_{1}, \ldots, a_{m}\right) \in A^{m+1}: \mathfrak{A} \models \theta_{i}[\mathbf{a}]\right\} .
$$

Over these expansions

$$
\varphi(X, x) \equiv \bigwedge_{i=1}^{k} \forall \mathbf{y}\left(R_{i} x \mathbf{y} \rightarrow \delta(X, \mathbf{y})\right) .
$$

\subsection{Unfoldings and tree representations}

The following technique of switching between tree representations and relational structures is similar to the one used in [10].

Let the vocabulary of $\varphi$ be $\tau$, and $\tau^{\theta}$ its extension by the $R_{i}$ as introduced above. For tree representations of $\tau^{\theta}$ structures we use a vocabulary $\sigma\left[\tau^{\theta}\right]$ consisting of the following unary and binary predicates:

- for each $\tau^{\theta}$-structure $\mathfrak{B}$ of size up to $m+1$ and an enumeration of these elements as $\mathbf{b}=\left(b_{0}, \ldots, b_{m}\right)$, a unary predicate $P_{\beta}$ associated with the isomorphism type $\beta$ of $(\mathfrak{B}, \mathbf{b})$ (or, equivalently, the quantifier-free type of $\mathbf{b}$ in $\mathfrak{B})$;

- for each $\rho \subseteq\{0, \ldots, m\} \times\{0, \ldots, m\}$ that is the graph of a partial function, a binary relation $E_{\rho}$. We include $\rho=\emptyset$.

With $\mathfrak{A}^{\theta}$ and a suitable tuple $\mathbf{a} \in A^{m+1}$ we associate

- a transition system $K\left(\mathfrak{A}^{\theta}\right)$ of type $\sigma\left[\tau^{\theta}\right]$, with distinguished node $\lambda=\mathbf{a}$,

- the tree unfolding $T\left(\mathfrak{A}^{\theta}, \mathbf{a}\right)$ of $K\left(\mathfrak{A}^{\theta}\right)$, which is a $\sigma\left[\tau^{\theta}\right]$ tree structure with root $\lambda=\mathbf{a}$. 
Conversely, we associate with every $\sigma\left[\tau^{\theta}\right]$ tree structure $T$ satisfying some weak FO definable consistency conditions outlined below:

- a tree-like $\tau^{\theta}$-structure $\mathfrak{B}(T)$, of which $T$ is a tree decomposition.

In particular, the tree unfolding of $\mathfrak{A}^{\theta}$ from a:

- $\left(\mathfrak{A}^{\theta}\right)^{*}:=\mathfrak{B}\left(T\left(\mathfrak{A}^{\theta}, \mathbf{a}\right)\right)$.

The transition system: The nodes of $K\left(\mathfrak{A}^{\theta}\right)$ are those $(m+1)$-tuples of $\mathfrak{A}^{\theta}$ that are in one of the relations $R_{i}$ (i.e., that satisfy one of the $\left.\theta_{i}\right)$, plus all $(m+1)$-tuples of the form $(a, \ldots, a)$ representing single elements. A node $\mathbf{a}$ is in $P_{\beta}$, if $\beta$ is the quantifier-free type of $\mathbf{a}$ in $\mathfrak{A}^{\theta}$. Transitions are labelled with labels $\rho$ to indicate identities between components of different tuples. $\left(\mathbf{a}, \mathbf{a}^{\prime}\right) \in E_{\rho}$ for $\rho=\left\{(i, j): a_{i}=a_{j}^{\prime}\right\}$. Note that $\rho=\emptyset$ is admitted, and applies to any two nodes corresponding to disjoint tuples; between any two nodes of $K\left(\mathfrak{A}^{\theta}\right)$ there is a transition.

The trees: $\quad T\left(\mathfrak{A}^{\theta}, \mathbf{a}\right)$ is the usual bisimilar unfolding of $K\left(\mathfrak{A}^{\theta}\right)$ into a tree structure, rooted in node $\mathbf{a}$.

Let $\mathcal{C}^{*}=\left\{T\left(\mathfrak{A}^{\theta}, \mathbf{a}\right): \mathfrak{A}\right.$ a $\tau$-structure $\}$ be the class of all $\sigma\left[\tau^{\theta}\right]$ trees obtained in this manner.

All $T \in \mathcal{C}^{*}$ satisfy some local and FO definable consistency criteria Cons related to the node labels and transition labels. These enforce, for $\left(u, u^{\prime}\right) \in E_{\rho}, u \in P_{\beta}$, $u^{\prime} \in P_{\beta^{\prime}}$, the obvious local compatibility conditions between the quantifier-free types $\beta$ and $\beta^{\prime}$ w.r.t. the identification encoded in $\rho$. For instance, for $\rho$ such that $i_{1}, \ldots, i_{k} \in$ $\operatorname{dom}(\rho)$, the following is in Cons:

$$
\forall y_{1} \forall y_{2}\left(E_{\rho} y_{1} y_{2} \rightarrow \bigvee \Phi\left(y_{1}, y_{2}\right)\right)
$$

where $\Phi$ is the set of all formulae $P_{\beta} y_{1} \wedge P_{\beta^{\prime}} y_{2}$ for pairs $\beta, \beta^{\prime}$ for which $\rho: \beta\left\lceil\left\{i_{1}, \ldots, i_{k}\right\} \simeq \beta^{\prime} \uparrow\left\{\rho\left(i_{1}\right), \ldots, \rho\left(i_{k}\right)\right\}\right.$.

The class $\mathcal{C}^{*}$, however, is not even MSO definable. A crucial property of $T \in \mathcal{C}^{*}$ is their homogeneity which stems from the fact that in $K\left(\mathfrak{A}^{\theta}\right)$ every node is reachable from every other one. For the tree unfolding this implies that the sibling subtrees at any two nodes are set-wise isomorphic.

Relational structures from trees: With every tree $T \models$ Cons associate a relational structure $\mathfrak{B}(T)$ as follows. Each node $u \in P_{\beta}$ of $T$ gives rise to a relational structure $\mathfrak{B}_{u}$ of size up to $(m+1)$ according to the isomorphism type encoded in $\beta . \mathfrak{B}(T)$ is obtained from the disjoint union of the $\mathfrak{B}_{u}$ for $u \in T$, with identifications according to $\rho$ between $\mathfrak{B}_{u}$ and $\mathfrak{B}_{u^{\prime}}$ for $\left(u, u^{\prime}\right) \in E_{\rho}$. More formally, let $(u, i) \in T \times\{0, \ldots, m\}$ be the element of the disjoint union of the $\mathfrak{B}_{u}$ that corresponds to the $i$-th element of $\mathfrak{B}_{u}$. Let then $\approx$ be the symmetric and transitive closure of the binary relation consisting of the pairs $\left((u, i),\left(u^{\prime}, i^{\prime}\right)\right)$ for $\left(u, u^{\prime}\right) \in E_{\rho}$ and $\left(i, i^{\prime}\right) \in \rho$. The universe of $\mathfrak{B}(T)$ is the quotient of the disjoint union of the $\mathfrak{B}_{u}$ w.r.t. $\approx$. Cons is such that $\approx$ is compatible with the relational information in the individual $\mathfrak{B}_{u}$.

It is important to note that the interpretation of the relations $R_{i}$ over $\mathfrak{B}(T)$ is not in general the one that would be defined by the $\theta_{i}$ over the $\tau$-reduct of $\mathfrak{B}(T)$. In general one only has that

$$
(*) \quad \mathfrak{B}(T) \models \forall \mathbf{z}\left(R_{i} \mathbf{z} \rightarrow \theta_{i}(\mathbf{z})\right),
$$

due to positivity of the $\theta_{i}$ and the construction of $\mathfrak{B}(T)$.

Remark: $T$ provides a tree decomposition of width $m$ of $\mathfrak{B}(T)$; the patches of this tree decomposition are guarded in the extended vocabulary $\tau^{\theta}$.

For a tree unfolding $\left(\mathfrak{A}^{\theta}\right)^{*}:=\mathfrak{B}\left(T\left(\mathfrak{A}^{\theta}, \mathbf{a}\right)\right)$ let $\pi:\left(\mathfrak{A}^{\theta}\right)^{*} \rightarrow \mathfrak{A}^{\theta}$ be the natural projection.

We denote as $\mathfrak{A}^{*}$ the $\tau$-reduct of $\left(\mathfrak{A}^{\theta}\right)^{*}$.

Lemma 17. $\pi:\left(\mathfrak{A}^{\theta}\right)^{*} \rightarrow \mathfrak{A}^{\theta}$ preserves the stages of the fixed point generation for $\varphi$ in the sense that for all $\alpha$ : $X^{\alpha}\left[\mathfrak{A}^{*}\right]=\pi^{-1}\left(X^{\alpha}[\mathfrak{A}]\right)$.

Proof. The claim is clear for $\alpha=0$; also limit stages are trivial. So consider a successor step. Let $\pi\left(c^{*}\right)=c$. We show that

$$
\mathfrak{A}^{*} \models \varphi\left[X^{\alpha}\left[\mathfrak{A}^{*}\right], c^{*}\right] \quad \text { iff } \quad \mathfrak{A} \models \varphi\left[X^{\alpha}[\mathfrak{A}], c\right],
$$

assuming that $X^{\alpha}\left[\mathfrak{A}^{*}\right]=\pi^{-1}\left(X^{\alpha}[\mathfrak{A}]\right)$.

Let $\mathfrak{A}^{*} \models \varphi\left[X^{\alpha}, c^{*}\right]$ and let $\mathfrak{A}=\theta_{i}[c, \mathbf{c}]$. By construction of $\mathfrak{A}^{*}$ there is $\mathbf{c}^{*} \in \pi^{-1}(\mathbf{c})$ such that $\mathfrak{A}^{*} \models \theta_{i}\left[c^{*}, \mathbf{c}^{*}\right]$. So $\mathfrak{A}^{*} \models \delta\left[X^{\alpha}, \mathbf{c}^{*}\right]$ and hence $\mathfrak{A} \models \delta\left[X^{\alpha}, \mathbf{c}\right]$. It follows that $\mathfrak{A}=\varphi\left[X^{\alpha}, c\right]$.

Conversely, let $\mathfrak{A}=\varphi\left[X^{\alpha}, c\right]$ and $\mathfrak{A}^{*} \models \theta_{i}\left[c^{*}, \mathbf{c}^{*}\right]$. Then $\mathfrak{A}=\theta_{i}[c, \mathbf{c}]$ for $\mathbf{c}:=\pi\left(\mathbf{c}^{*}\right)$, as $\pi$ is a homomorphism. It follows that $\mathfrak{A}=\delta\left[X^{\alpha}, \mathbf{c}\right]$ and thus $\mathfrak{A}^{*}=\delta\left[X^{\alpha}, \mathbf{c}^{*}\right]$ by the inductive hypothesis. So $\mathfrak{A}^{*} \models \varphi\left[X^{\alpha}, c^{*}\right]$.

\subsection{A translation into modal logic}

We want to capture the generation of the least fixed point of $\varphi$ over $\mathfrak{A}^{*}$ in terms of the associated tree $T\left(\mathfrak{A}^{\theta}, \mathbf{a}\right)$. A modal system $\bar{\varphi}(\bar{X})$ for $\bar{X}=\left(X_{0}, \ldots, X_{m}\right)$ can serve this purpose. A system rather than recursion in a single predicate variable $X$ is necessary, as each node in the tree stands for an $(m+1)$-tuple. The intended meaning of $u \in X_{j}^{\alpha}$ will be that the $j$-th component of this tuple is in $X^{\alpha}$. Correspondingly, $\bar{\varphi}$ is of the form $\bar{\varphi}(\bar{X})=$ $\left(\varphi_{0}(\bar{X}), \ldots, \varphi_{m}(\bar{X})\right)$.

Recall that $\varphi(X, x)$ is the conjunction of the following, for $1 \leqslant i \leqslant k$ :

$$
\forall y_{1} \ldots \forall y_{m}\left(\theta_{i}\left(x, y_{1}, \ldots, y_{m}\right) \rightarrow \delta\left(X, y_{1}, \ldots, y_{m}\right)\right)
$$


$\varphi_{j}(\bar{X})$ has, for each $1 \leqslant i \leqslant k$ and each quantifier-free $\tau^{\theta}$-type $\beta=\beta\left(x_{0}, \ldots, x_{m}\right)$ such that $\beta \models \theta_{i}$ the conjunct

$$
\bigwedge_{\rho(j)=0}\left[E_{\rho}\right]\left(P_{\beta} \rightarrow \delta(\bar{X})\right)
$$

where $\delta(\bar{X})$ is the disjunction of those $X_{s}$ for which $X y_{s}$ is a disjunct in $\delta(X, \mathbf{y})$.

The following correspondence between $\varphi$ and $\bar{\varphi}$ is based on a correspondence between the individual stages, using Lemma 17.

Recall that $\mathcal{C}^{*}$ is the class of tree structures $T\left(\mathfrak{A}^{\theta}, \mathbf{a}\right)$ that represent unfoldings $\mathfrak{A}^{*}$ of $\tau$-structures $\mathfrak{A}$. With a node $u$ of $T\left(\mathfrak{A}^{\theta}, \mathbf{a}\right)$ we associate the tuple $\mathbf{b}=\pi(u) \in A^{m+1}$ : this is the tuple $\mathbf{b}$ which, as an element of $K\left(\mathfrak{A}^{\theta}\right)$, gives rise to $u$ in the tree unfolding, or the last node in the path $u$. We also denote the $j$-th component $b_{j}$ of this tuple $\mathbf{b}$ by $\pi_{j}(u)$, thus regarding $\pi_{j}$ as a map $\pi_{j}: T\left(\mathfrak{A}^{\theta}, \mathbf{a}\right) \rightarrow A$ for $j \leqslant m$.

Lemma 18. Consider the stages $\left(X_{j}^{\alpha}\right)_{j \leqslant m}$ of the fixed point generation for system $\bar{\varphi}$ over $T\left(\mathfrak{A}^{\theta}, \mathbf{a}\right)$ and the stages $X^{\alpha}$ in the generation of the fixed point for $\varphi$ over $\mathfrak{A}$. Then, for all $\alpha$, the $\left(X_{j}^{\alpha}\right)$ represent $X^{\alpha}$ :

$$
X_{j}^{\alpha}=\left\{u \in T\left(\mathfrak{A}^{\theta}, \mathbf{a}\right): \pi_{j}(u) \in X^{\alpha}\right\} .
$$

It follows that $\varphi$ is bounded iff $\bar{\varphi}$ is bounded over $\mathcal{C}^{*}$.

Proof. As $\bar{\varphi}$ is modal, and since $T\left(\mathfrak{A}^{\theta}, \mathbf{a}\right)=T\left(K\left(\mathfrak{A}^{\theta}\right), \mathbf{a}\right)$ is bisimilar to $K\left(\mathfrak{A}^{\theta}\right)$, we may prove the correspondence between $\varphi$ on $\mathfrak{A}$ and $\bar{\varphi}$ on $K\left(\mathfrak{A}^{\theta}\right)$, where is becomes:

$$
X_{j}^{\alpha}=\left\{\mathbf{b} \in K\left(\mathfrak{A}^{\theta}\right): b_{j} \in X^{\alpha}\right\} .
$$

We write $K$ for $K=K\left(\mathfrak{A}^{\theta}\right)$ for the rest of this proof.

The claim for the stages $\left(X_{j}^{\alpha}\right)_{j \leqslant m}$ of $\bar{\varphi}$ over $K$ and the stages $X^{\alpha}$ of $\varphi$ over $\mathfrak{A}$ is proved by induction on $\alpha$. Consider the successor step from $\alpha$ to $\alpha+1$ and assume the claim for $\alpha$.

Let $\mathbf{b} \in X_{j}^{\alpha+1}$, i.e., $K, \mathbf{b} \models \varphi_{j}\left[\bar{X}^{\alpha}\right]$. To show that $b_{j} \in X^{\alpha+1}$ in $\mathfrak{A}$, we need to establish that, for all $\mathbf{b}^{\prime}=$ $\left(b_{j}, b_{1}^{\prime}, \ldots, b_{m}^{\prime}\right)$ such that $\mathfrak{A} \models \theta_{i}\left[\mathbf{b}^{\prime}\right]$ also $\mathfrak{A}, \mathbf{b}^{\prime} \models \delta\left[X^{\alpha}\right]$. $\mathfrak{A} \models \theta_{i}\left[\mathbf{b}^{\prime}\right]$ implies that $\mathbf{b}^{\prime}$ is a node of $K$, contained in some $P_{\beta}$ for which $\beta \models \theta_{i}$, and $\left(\mathbf{b}, \mathbf{b}^{\prime}\right) \in E_{\rho}$ for some $\rho$ with $\rho(j)=0$. For these $\beta$ and $\rho$, therefore, $\varphi_{j}$ has the conjunct $\left[E_{\rho}\right]\left(P_{\beta} \rightarrow \delta(\bar{X})\right)$, and $K, \mathbf{b} \models \varphi_{j}\left[\bar{X}^{\alpha}\right]$ implies that $K, \mathbf{b}^{\prime} \models \delta\left[\bar{X}^{\alpha}\right]$. It follows that $\mathfrak{A}, \mathbf{b}^{\prime} \models \delta\left[X^{\alpha}\right]$.

Conversely, let $\mathbf{b} \in K$ and $b_{j} \in X^{\alpha+1}$. Then $\mathfrak{A} \models$ $\varphi\left[X^{\alpha}, b_{j}\right]$ implies that $\mathbf{b} \in X_{j}^{\alpha+1}$ as follows. Consider a conjunct $\left[E_{\rho}\right]\left(P_{\beta} \rightarrow \delta(\bar{X})\right)$ in $\varphi_{j}$, where $\beta \models \theta_{i}$. Then $\left(\mathbf{b}, \mathbf{b}^{\prime}\right) \in E_{\rho}$ implies that $b_{j}=b_{0}^{\prime}$, and $\mathbf{b}^{\prime} \in P_{\beta}$ implies that $\mathfrak{A} \models \theta_{i}\left[\mathbf{b}^{\prime}\right]$. Therefore, $\mathfrak{A} \models \varphi\left[X^{\alpha}, b_{j}\right]$ implies, as $\mathfrak{A} \models$ $\theta_{i}\left[\mathbf{b}^{\prime}\right]$, that $\mathfrak{A}=\delta\left[X^{\alpha}, \mathbf{b}^{\prime}\right]$. Therefore $K, \mathbf{b}^{\prime}=\delta\left[\bar{X}^{\alpha}\right]$. We thus get $K, \mathbf{b} \models \varphi_{j}\left[\bar{X}^{\alpha}\right]$ and $\mathbf{b} \in X_{j}^{\alpha+1}$.
This reduction, however, does not yield decidability directly as the class $\mathcal{C}^{*}$ of relevant tree structures is not MSO definable. We must therefore extend the class $\mathcal{C}^{*}$ of actual tree representations to a wider class $\mathcal{C} \supseteq \mathcal{C}^{*}$ that is MSO definable and still supports a correspondence as in the lemma. The lemma implies

$$
\varphi \text { unbounded } \Rightarrow \bar{\varphi} \text { unbounded over } \mathcal{C}
$$

for any $\mathcal{C} \supseteq \mathcal{C}^{*}$. Crucial for the choice of $\mathcal{C}$ is that it is tight enough to make the converse true as well.

\subsection{Monotonicity and admissible trees}

Consider an arbitrary tree $T \models$ Cons and the associated $\tau$-structure $\mathfrak{B}(T)$ represented by $T$. It is clear from the construction of $\mathfrak{B}(T)$ that there is a translation from MSO over $\mathfrak{B}(T)$ into MSO over $T$. Essentially this amounts to a translation $\psi \mapsto \hat{\psi}$ such that $\mathfrak{B}(T)=\psi \Leftrightarrow T=\hat{\psi}$. Some care has to be taken w.r.t. first- and second-order free variables, though. Consider for instance a formula $\psi(X, x)$. An instantiation by $P \subseteq \mathfrak{B}(T)$ is represented in $T$ by a tuple $\bar{P}=\left(P_{0}, \ldots, P_{m}\right)$ of subsets of $T$, where

$$
P_{j}=\left\{u \in T: u_{j} \in P\right\} \subseteq T,
$$

$u_{j}$ the $j$-th component of the tuple represented in $\mathfrak{B}_{u}$. Similarly, w.r.t. its first-order variable $x, \psi$ translates into a tuple of formulae $\hat{\psi}_{j}$ such that $\hat{\psi}_{j}$ expresses of $u \in T$ whether $\psi$ is true of $u_{j}$.

So in this case (with just one free variable of each type), $\hat{\psi}$ is of the form $\left(\hat{\psi}_{j}(\bar{X}, y)\right)_{0 \leqslant j \leqslant m}$ and such that for all $j$ :

$$
\mathfrak{B}(T) \models \psi\left[P, u_{j}\right] \quad \text { iff } \quad T \models \hat{\psi}_{j}[\bar{P}, u] .
$$

The availability of MSO quantification in this translation is essential even for the translation of FO formulae $\psi$, since the set of nodes of $T$ in which a given element of $\mathfrak{B}(T)$ is represented can only be defined in MSO. This is because the same element can be carried along $\left(E_{\rho}\right)$-paths of arbitrary lengths. For instance, in order to check whether $\psi(x)=\exists y R x y$ is true of some $x$, which is represented as the $i$-th component of $u \in T, \hat{\psi}_{i}$ needs to involve checking all nodes $u^{\prime}$ reachable from $u$ on paths labelled $\rho_{1} \ldots \rho_{k}$ such that $i \in \operatorname{dom}\left(\rho_{k} \circ \cdots \circ \rho_{1}\right)$.

Lemma 19. There is an effective translation of $\varphi(X, x)$ into $\left(\hat{\varphi}_{j}(\bar{X}, x)\right)_{0 \leqslant j \leqslant m}$ such that for all $P \subseteq \mathfrak{B}(T)$, represented by $\bar{P}$ over $T$, and for $0 \leqslant j \leqslant m$ :

$$
\mathfrak{B}(T) \models \varphi\left[P, u_{j}\right] \quad \text { iff } \quad T \models \hat{\varphi}_{j}[\bar{P}, u] .
$$

Similarly, for the fixed points:

$$
\mathfrak{B}(T) \models \varphi^{\infty}\left[u_{j}\right] \quad \text { iff } \quad T \models(\hat{\varphi})_{j}^{\infty}[u] .
$$


Due to (negative) monotonicity of $\varphi$ in the non-recursive predicates, the evaluation of the fixed point w.r.t. $\bar{\varphi}$ in $T$ will in general result in an overestimate for the fixed point of $\varphi$ over $\mathfrak{B}(T)$. More generally, for any $P \subseteq \mathfrak{B}(T)$ and its representation $\bar{P}$ over $T$, by (*) in section 5.1 ,

$$
(* *) \quad T \models \forall x\left(\hat{\varphi}_{j}(\bar{X}, x) \rightarrow(\bar{\varphi})_{j}(\bar{X}, x)\right) .
$$

Definition 20. Call a tree $T$ admissible for $\varphi$ if $T$ is consistent (cf. local FO conditions Cons) and $\hat{\varphi}_{j}^{\infty}=\bar{\varphi}_{j}^{\infty}$ on $T$ for $j=0, \ldots, m$. Let $\mathcal{C}_{\varphi}$ be the class of trees that are admissible for $\varphi$.

Observation 21. $\mathcal{C}_{\varphi}$ is $\mathrm{MSO}$ definable.

Lemma 22. $\varphi$ is bounded iff $\bar{\varphi}$ is bounded over $\mathcal{C}_{\varphi}$.

Proof. $\mathcal{C}_{\varphi} \supseteq \mathcal{C}^{*}$ follows from Lemma 18 . The tree structure $T\left(\mathfrak{A}^{\theta}, \mathbf{a}\right)$ underlying $\mathfrak{A}^{*}$ provides a faithful representation of the fixed point generation of $\varphi$ (over $\mathfrak{A}$ or $\mathfrak{A}^{*}$, see Lemma 17) in terms of $\bar{\varphi}$. It follows that $\bar{\varphi}$ is unbounded over $\mathcal{C}_{\varphi}$ if $\varphi$ is unbounded.

Consider $\varphi$ that is bounded by $n \in \mathbb{N}$. By the definition of $\mathcal{C}_{\varphi}$, the results of the fixed points w.r.t. $\bar{\varphi}$ and the faithful translation $\hat{\varphi}$ of $\varphi$ on $T \in \mathcal{C}_{\varphi}$ are the same. By monotonicity $(* *), \bar{\varphi}$ can only overestimate individual stages, which means that $\gamma[\bar{\varphi}, T] \leqslant \gamma[\hat{\varphi}, T]=\gamma[\varphi, \mathfrak{B}(T)] \leqslant n$. So $\bar{\varphi}$ is bounded over $\mathcal{C}_{\varphi}$.

This finishes the proof of the main theorem, since boundedness of the modal system $\bar{\varphi}$ over the MSO definable class $\mathcal{C}_{\varphi}$ is decidable by Theorem 14 .

\section{Summary}

Decidability of $\operatorname{Bdd}(\mathcal{F}(\forall,-))$ has been shown by model theoretic methods, involving a reduction to the boundedness problem for modal systems over some suitable MSO definable class of trees. Both the modal system and the class over which its boundedness is checked depend on the input formula $\varphi(X, x) \in \mathcal{F}(\forall,-)$, and the ultimate target in the reduction is a satisfiability issue in the MSO theory of the $\omega$-branching tree.

As a result of this approach we cannot expect to extract good complexity bounds. The complexity of $\operatorname{Bdd}(\mathcal{F}(\forall,-))$ remains to be determined.

Applicability of the reduction seems to depend, in a rather subtle way, on the very special monotonicity properties involved in universal quantifications in which the only role that the (exclusively negative) occurrences of nonrecursive predicates play is that of restricting the scope of the universal quantifiers, as in universal guarded quantification.
Among the obvious ramifications, therefore, that can be treated in an analogous fashion are systems of $\mathcal{F}(\forall,-)$ formulae in monadic recursive predicates, and universal formulae of the guarded fragment in arbitrary arities.

It remains open whether binary purely universal recursion - with otherwise the same constraints on polarities and equality - has a decidable boundedness problem.

The present investigation covers recursion in monadic recursive predicates (or higher arity but then guarded universal rather than arbitrary universal quantification would seem to be essential).

In the wider picture, major open issues concern the boundedness problem for the full guarded fragment, as well as the monadic boundedness problem for larger fragments or all of FO on the class of structures of tree-width $k$, for fixed $k$. In both cases, suitable reductions to the MSO theory of trees could possibly provide a route to decidability proofs.

\section{References}

[1] S. Abiteboul, R. Hull, and V. Vianu. Foundations of Databases. Addison-Wesley, 1995.

[2] M. Ajtai and Y. Gurevich. Datalog vs. first-order logic. Journal of Computer and System Sciences, 49, pages 562-588, 1994.

[3] H. Andréka, J. van Benthem, and I. Németi. Modal languages and bounded fragments of predicate logic. Journal of Philosophical Logic, 27, pages 217-274, 1998.

[4] A. Atserias, A. Dawar, and P. Kolaitis. On preservation under homomorphisms and unions of conjunctive queries. In Proc. 23rd Symp. on Principles of Database Systems, pages 319-329, 2004.

[5] J. Barwise and Y. Moschovakis. Global inductive definability. Journal of Symbolic Logic, 43(3), pages 521-534, 1978.

[6] J. van Benthem. Modal Logic and Classical Logic. Bibliopolis, Naples, 1985.

[7] R. Berger. The Undecidability of the Domino Problem, volume 66 of Memoirs of the AMS. American Mathematical Society, 1966.

[8] E. Börger, E. Grädel, and Y. Gurevich. The Classical Decision Problem. Springer-Verlag, 1997.

[9] S. Cosmadakis, H. Gaifman, P. Kanellakis, and M. Vardi. Decidable optimization problems for database logic programs. In Proc. 20th ACM Symp. on Theory of Computing, pages 477-490, 1988. 
[10] E. Grädel, C. Hirsch, and M. Otto. Back and forth between guarded and modal logics. ACM Transactions on Computational Logic, 3, pages 418-463, 2002.

[11] H. Gaifman, H. Mairson, Y. Sagiv, and M. Vardi. Undecidable optimization problems for database logic problems. Journal of the ACM, 40(3), pages 683-713, 1993.

[12] G. Hillebrand, P. Kanellakis, H. Mairson, and M. Vardi. Undecidable boundedness problems for datalog programs. Journal of Logic Programming, 25(2), pages 163-190, 1995.

[13] P. Kanellakis. Elements of Relational Database Theory. In J. van Leeuwen, editor, Handbook of Theoretical Computer Science, volume B, pages 1073-1157. MIT Press/Elsevier, 1990.

[14] P. Kolaitis and M. Otto. On the boundedness problem for two-variable first-order logic. In Proc. 13th IEEE Symp. on Logic in Computer Science, pages 513-524, 1998.

[15] P. Kolaitis and M. Otto. On the boundedness problem for fragments of first-order logic: Undecidability results. unpublished draft.
[16] P. Kolaitis and C. Papadimitriou. Some computational aspects of circumscription. Journal of the ACM, 37, pages 1-14, 1990.

[17] J. McCarthy. Circumscription - a form of nonmonotonic reasoning. Artificial Intelligence, 13, pages 2739, 1980.

[18] Y. Moschovakis. Elementary Induction on Abstract Structures. North Holland, 1974.

[19] M. Otto. Eliminating recursion in the $\mu$-calculus. In Proc. 16th Symp. on Theoretical Aspects of Computer Science, volume 1563 of LNCS, pages 531-540, 1999.

[20] M. Otto. Modal and guarded characterisation theorems over finite transition systems. Annals of Pure and Applied Logic, 130, pages 173-205, 2004.

[21] B. Rossmann. Existential positive types and preservation under homomorphisms. In Proc. 20th IEEE Symp. on Logic in Computer, pages 467-476, 2005. 